\title{
The Design and Implementation of Documents System for Law Application

\author{
ZHAO Yan ${ }^{1, \text { a }}$ \\ ${ }^{1}$ Shaanxi vocational and technical college, Xi'an 710100,China \\ azhaoyao125@126.com
}

Keywords: XML Technology; Documents System; Public Security Organ

\begin{abstract}
Along with the successful application of office automation technology in the public security system, the traditional office mode gradually is digital, networked replaced by way of the modern office. Through the analysis of the content and format of legal documents, we can find the rule of legal documents, according to these rules, combined with the principle of database, we designed and developed a set of specially designed based on XML technology legal document production and management of the computer software system, the software system design, functional design, database and interface design, development of the system management, case management, document management, criminal suspects, such as function modules. The legal document system has the characteristics of advanced, easy to use, fully functional, greatly improving the production efficiency and fairness of the legal documents.
\end{abstract}

\section{Introduction}

With the successful application of office automation technology in the public security system, the traditional office mode gradually is digital, networked replaced by way of the modern office. Document writing is the important content in administrative work, the public security organ is the legal document used by the public security organs in criminal law enforcement has the force of law in the routine paperwork, it is serious, a fixed structure, adopt the stylized prose, set requires accurate language specification, explain a single, concise, easy to understand, must not be ambiguous, specious, so the legal document drafted use is usually special laws trained legal workers to qualify [1]. In recent years, with the popularization of computer, the police documents basically made by the electronic mode, by the way of handwritten improvement for the computer to print way, drafting and editing is mainly through the office software such as word, WPS [2].

Practice, instrument making is quite a lot of work, due to some clerical work experience of authors and writing level is not enough, on the format of the document, writing ability is not strong, the document quality is low, serious and even affected the legal effect of the documents, to the progress of the criminal litigation has created nothing but trouble. With the development of computer application technology in the public security industry to raise the level of information management and case we consider using the computer software application system to achieve production and management of documents.

\section{XML technology overview}

XML shall be formulated by the W3C, as a new technology of the Internet, XML has a very wide range of applications, and XML has penetrated the various application fields. Extensible Markup Language Markup Language) is a subset of XML to describe a class called XML file data object, also part description of the behavior of the computer program processing these data objects.XML is a concrete application of SGML or restricted form. From the point of structure, the XML file is formal SGML files. Its aim is to make on the Web to existing Hypertext Markup Language HTML (Hypertext Markup Language) way to provide, receiving and processing generic SGML is possible.XML design is considered the ease of implementation, but also take into account the interoperability with both SGML and HTML [3-4]. The application of XML technology is more and more widely, emerge in endlessly, new achievements in the application has been clear about the 
new requirements, new technology, a new theory. Following a brief introduction of the XML technology system, the W3C groups such as research and development of XML technology system block diagram of system is shown in figure 1 .

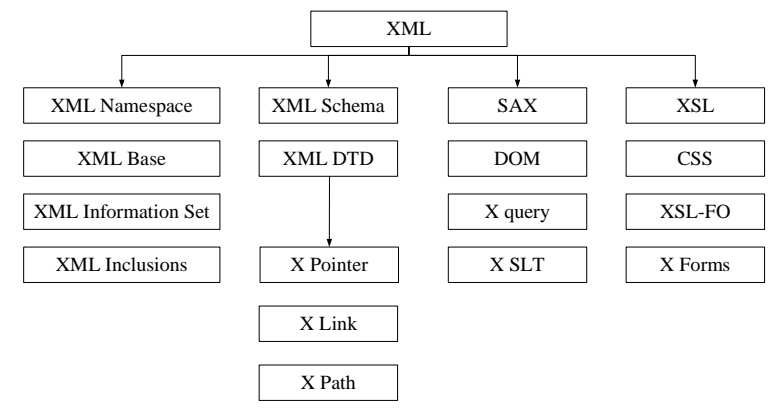

Figure 1. The structure of XML technology

\section{The legal document system requirements analysis}

The legal document refers to the public security organs in handling criminal cases that have adopted by the force of law in the process of programming documents. In the practical work, a lot of documents cannot meet the format of the specification requirements, some even affected the documents of legal effect. With the development of computer application technology, the use of the software system to be solve the problem of documents [5]. Application of computer software system can improve the efficiency of document production. By analyzing the format and characteristics of each instrument, create a document template scientifically, establish case information database, to avoid repeating entry and paperwork to fill out the information of the non-standard problem, the function of the system structure is shown in figure 2.

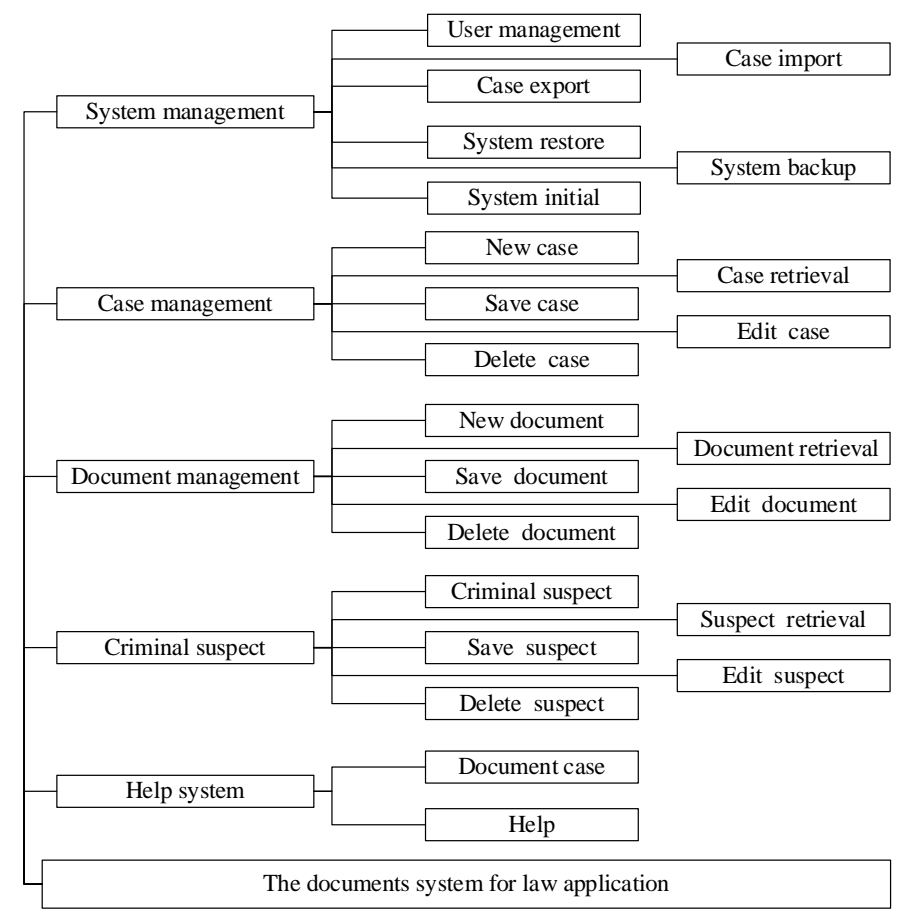

Figure 2. The function module of documents system for law application

Through the system can improve the document normative legal document. Current handmade papers, the investigators write different, paper quality is uneven. Software system design to compare with the related regulations, to avoid some of the workers take it for granted documents method and the artificial operation, ensure the normative documents.

Through the legal document system can improve the level of case information management. The public security organ is a legal document for the criminal cases handled by the public security organ 
and the regulatory activity of the product, is also a true record of the case and regulatory activities, in the process of investigation of the case, start to accept the case to the investigation of the end of each program and each activity, with a legal document [1].Documents to make use of the software system record is equivalent to the whole process of the case, through which can intuitive understanding to the progress of each case, criminal suspects, sending information such as investigation situation, decision making for policy makers to provide important basis.

The legal document management system to drive of the application of computer technology application in other public security work. Legal document is an important part of the public security work and the most common form of job content, is the link between the processes of case. Legal documents of the electronic and intelligent is the basis of the implementation of public security office automation system, application of legal document management system can make more public security workers realize the advantages of science and technology, it is bound to promote the application of computer technology in other public security work.

\section{The design of documents system for law application}

As a legal document production tools, the main function of the system is based on computers replace manual, realize the intelligence of legal documents. The characteristics of the analysis instrument, the fixed language part of the document as a template, it made the spreadsheet software, users only need to fill in the personalized information as required. Using data correlation, any information need to fill in time, do not need to repeat to fill in, when a new document, the system automatically will have been transferred to fill in the information. For maximum flexibility, the implementation of the system generated document is WORD format document, allowing users to edit and print again the documents. This system is based on web way, using B/S structure, to achieve data centralized control, distributed access by the user. As shown in figure 3 is the system of the network topology structure.

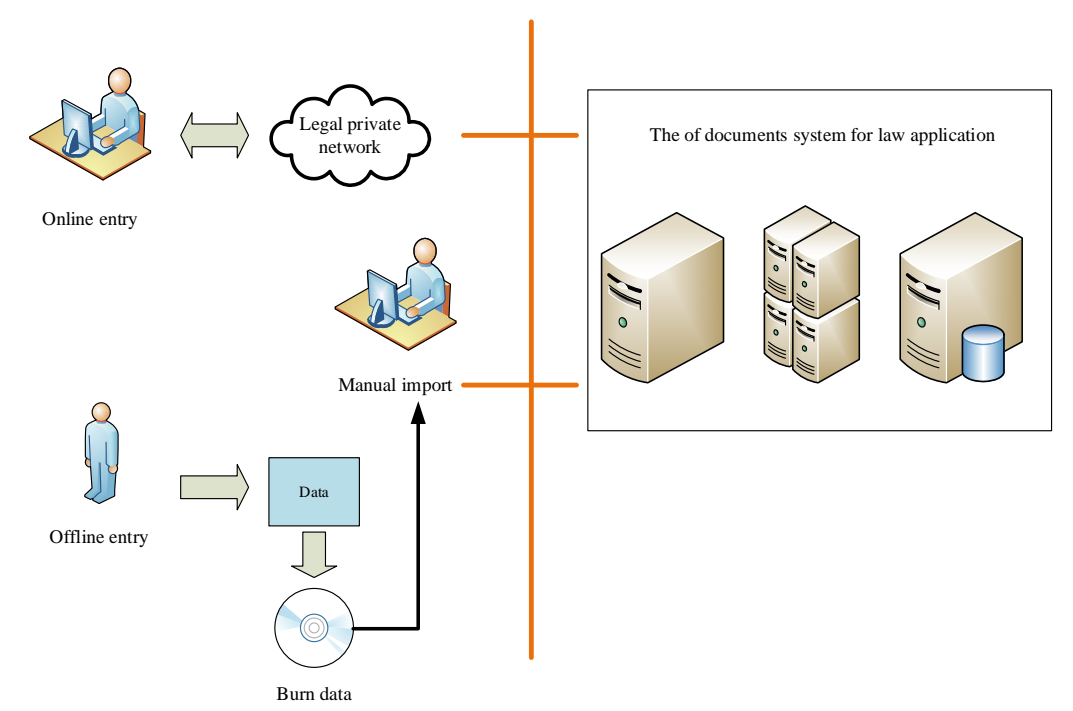

Figure 3. The structure of documents system for law application

On reasonable development $\mathrm{B} / \mathrm{S}$ architecture design online legal document (offline) management system, to provide a safe, reliable and high-speed data transmission and strong and flexible application platform, realize information needs of departments. Construction safety, stability and management system. The establishment of a flexible application service platform, the structured design of system structure, to achieve standardization application requirements; Through to the various functions for centralized, block, according to the requirements of structured programming, based on the characteristics of Marine administrative law enforcement work and the actual work environment, legal documents management system network topology structure is designed. 


\section{Conclusion}

This article from the system development background, the user demand investigation, demand analysis, the establishment of the design ideas, function module design and other aspects in detail the design process of the system. According to the characteristics of the instrument and the characteristics of computer database technology, analyzes the feasibility of this system, puts forward how to make use of computer software technology improve the efficiency of the public security organ legal document writing and normative method. Paper by analyzing the case of the public security organ case process and the structure characteristics of the criminal legal documents and production requirements, determine the document format requirements - document template method to realize the complete document. Paper based on the documents in the field of statistics and analysis, to determine the system data structure and database design of the basic scheme, at the same time, established the system of the development environment and application structure, for programmers to implement system laid a good foundation.

\section{References}

[1] Rao, Praveen, and Bongki Moon. "An internet-scale service for publishing and locating XML documents." Data Engineering, 2009. ICDE'09. IEEE 25th International Conference on. IEEE, 2009.

[2] Gerner, Martin, Goran Nenadic, and Casey M. Bergman. "LINNAEUS: a species name identification system for biomedical literature." BMC bioinformatics 11.1 (2010): 85.

[3] Berrick, Stephen W., et al. "Giovanni: a web service workflow-based data visualization and analysis system." Geoscience and Remote Sensing, IEEE Transactions on 47.1 (2009): 106-113.

[4] < div id="gs_cit0" tabindex="0" class="gs_citr"> Thuy, Pham Thi Thu, Young-Koo Lee, and SungYoung Lee. "DTD2OWL: automatic transforming XML documents into OWL ontology." $<\mathrm{i}>$ Proceedings of the 2nd International Conference on Interaction Sciences: Information Technology, Culture and Human $</ \mathrm{i}>$. ACM, 2009. $</$ div $>$

[5] Kwietniewski, Marcin, et al. "Transforming XML documents as schemas evolve."Proceedings of the VLDB Endowment 3.1-2 (2010): 1577-1580. 